\title{
Moralité anglaise et moralité européenne
}

Jean-Paul Debax

\section{(2) OpenEdition}

Journals

Édition électronique

URL : http://journals.openedition.org/shakespeare/1276

DOI : 10.4000/shakespeare.1276

ISSN : 2271-6424

Éditeur

Société Française Shakespeare

Édition imprimée

Date de publication : 1 novembre 1994

Pagination : 129-140

Référence électronique

Jean-Paul Debax, «Moralité anglaise et moralité européenne », Actes des congrès de la Société française Shakespeare [En ligne], 12 | 1994, mis en ligne le 01 janvier 2007, consulté le 25 avril 2019. URL : http://journals.openedition.org/shakespeare/1276; DOI : 10.4000/shakespeare.1276 
COSMOPOLITISME ET INSULARITÉ 


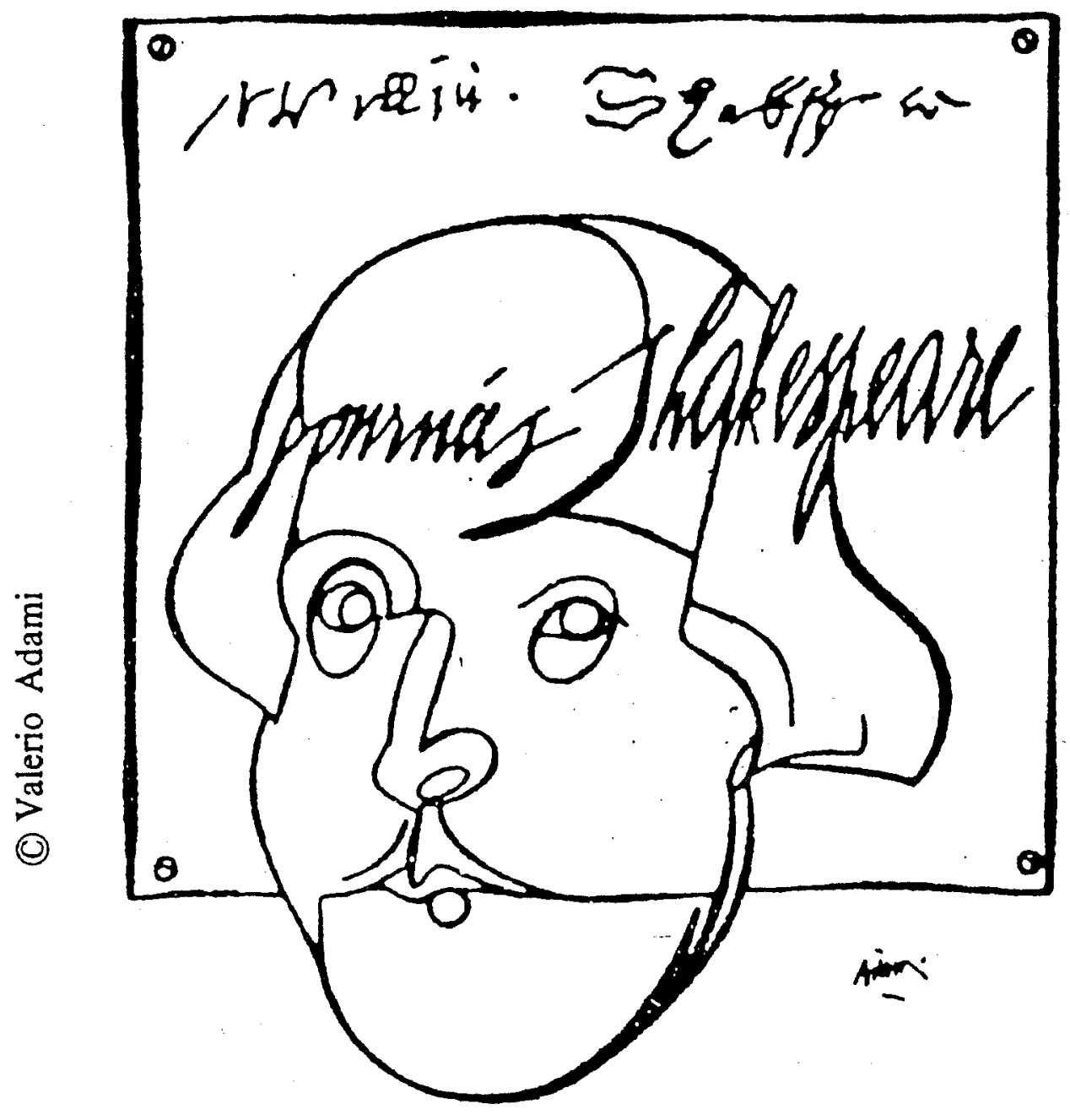

Affiche de Valerio Adami, spécialement conçue pour les journées Shakespeare 1979 


\section{SHAKESPEARE}

COSMOPOLITISME ET INSULARITÉ

Société Française Shakespeare

Actes du Congrès 1993

sous la direction

de

M. T. JONES-DAVIES

Ouvrage publié avec le soutien de

BARCLAYS

BARCLAYS BANK S.A

PARIS

LES BELLES LETTRES

1994 
Tous droits de traduction, de reproduction et d'adaptation réservés pour tous les pays.

(C) 1994 Société d'édition Les Belles Lettres, 95 bd Raspail 75006 Paris

ISBN 2.251.69122-7 


\section{Moralité anglaise et moralité européenne}

Ayant maintes fois récusé, dans le passé, le terme de "moralité", pour la raison qu'il ne s'appliquait à aucune production dans l'histoire du thêâtre médiéval tardif en Angleterre, et proposé à sa place l'appellation de "théâtre du Vice" pour décrire un corpus comprenant la majorité des pièces du $X^{e}$ et des trois premiers quarts du XVle siècle, je dois donc une explication aux lecteurs qui pourraient être surpris de trouver dans mon titre la conjonction des termes "moralite" et "anglaise" (conjonction qui n'est d'ailleurs qu'opératoire et temporaire, comme on le verra bientôt).

J'entendrai donc ici par "moralité", et à l'imitation de l'usage français des $\mathrm{XlV}^{\mathrm{e}}, \mathrm{XV}^{e}$ et $\mathrm{XVl}{ }^{\mathrm{e}}$ siècles, même si cet usage est lui-même, il faut l'avouer, passablement incertain, l'ensemble des pièces qui, d'une part, ne peuvent être définies comme des tropes liturgiques associés au rituel (comme les Quem Quaeritis ou les Nativités, en latin ou en langue vernaculaire), ni, d'autre part, comme des pièces neo-classiques, composées à partir de visées et de théories critiques a priori selon des principes puisés dans les œuvres littéraires et les écrits critiques de l'Antiquité.

L'opposition au XV1e siècle, entre théâtre "traditionnel" et théâtre neo-classique n'a pas besoin d'être démontrée. Les contemporains l'ont assez soulignée. Ainsi La Taille, dans sa préface à son Carrivaux (1574):

Et si on m'allègue qu'on joue ordinairement assez de jeux qui ont ce nom de comédies et tragédies, je leur rediray encore que ces beaux tiltres sont mal assortls à telles sottises, lesquelles ne retiennent rien de la façon ny du style des anciens.

Mais justement des anciens que faut-il retenir ? Sûrement pas les chœurs que le public boude :

En ceste tragédie, écrit Grévin dans le "Discours sur le théâtre" préfacé à sa Mort de César (1558), on trouvera par aventure estrange, que sans estre advoué d'aucun autheur ancien, j'ay faict la troupe interlocutaire de gensdarmes de bandes de César, et non de quelques chantres, ainsi qu'on a accoustumé ... J'ay en ceci esgard que je ne parloy pas aux Grecs, ny aux Romains, mais aux François, lesquels ne se plaisent pas beaucoup en ces 
chantres mal exercités, ainsi que j'ay souventesfois observé aux autres endroits où l'on en a mis en jeu.

Jean de Schelande est encore plus net, dans son Tyr et Sidon (1608) : «les choeurs ... sont tousjours désagréables, en quelque quantité ou qualité qu'ils paraissent». Lope de Vega a, de plus, la sagesse du réalisme, même si c'est sans enthousiasme. Dans son Arte nuevo de hacer comedias (1609), il se rend compte qu'il faut parler au peuple dans les formes que celui-ci attend :

porque como las paga el vulgo, es justo

hablarle en necio para darle gusto.

D'autre part, semble-t-il, le public se pressait en foule et avec enthousiasme aux Passions, Mystères, Moralités, Farces et Sotties, aux Autos Sacramentales espagnols, aux Sacre Representazione italiennes et aux Spelen van sinne des Pays Bas. Malgré la diversité indéniable de ces productions, elles ont bien des traits en commun et particulièrement un point de similarité dans leurs genèses respectives.

En effet, à la question : comment sont nés ces différents types de pièces, religieuses ou profanes, sérieuses ou comiques, ambitieuses ou modestes ? aucune réponse claire ne peut être apportée. L'histoire littéraire avance quelques suggestions, des filiations et la description de certaines évolutions. Mais ce dont on est sûr, négativement pour ainsi dire, c'est que ces œuvres n'ont pas fait l'objet d'une programmation concertée, pensée, prévue. En d'autres termes, elles sont le fruit d'une évolution naturelle, répondant sans doute essentiellement aux conditions historiques et culturelles de chaque période et à la demande d'un public potentiel. Autrement dit, cette production, même si certains courants s'y font jour, si certaines spécialisations s'y dessinent, ne connaît pas la fragmentation en genres, tels qu'ils seront entendus par les théoriciens neo-classiques. Et ce serait la pire des erreurs que de soumettre a posteriori ces pièces à un examen selon des critères reposant sur la distinction entre les genres tels qu'ils sont, par exemple, parodiquement évoqués dans les paroles de Polonius : «tragedy, comedy, history, pastoral, pastoral comical, historical pastoral, tragical-historical, tragical-comical-historical-pastoral». Halina Lewicka, pourtant orfevre on la matière, et à qui l'on doit plusieurs études remarquables sur le théâtre comique français et une impressionnante Bibliographie du Théâtre français des $X V^{e}$ et $X V I^{e}$ siècles, reconnaît que «vue la confusion des genres dramatiques au 
Moyen Age, tout choix est nécessairement subjectif», et Bernadette Rey-Flaud dans son étude sur la Farce, sous-titrée pourtant "Théorie d'une genre dramatique" renchérit : «le problème posé par la délimitation entre farce et sottie est plus ardu encore» ${ }^{1}$. En même temps que le concept donc, bannissons également le terme de "genre" au profit de mots comme "catégorie", "forme" ou "type".

Il ne faudrait pas supposer pour autant que cette indifférence vis a vis des catégories neo-classiques signifie une inorganisation totale, une anarchie généralisée ou une confusion pandémoniaque. Cette production connait au contraire des lignes de force, certes relativement souples et adaptables, et des modes différents qui se combinent relativement librement, en proportions variables selon les contenus et les occasions de représentation.

Revenant à notre thème, insularité et cosmopolitisme, je me propose dans un premier temps de montrer comment le théâtre d'époque Tudor participe des grands courants européens dans ses sujets, dans ses thèmes, et a été à maintes reprises influencé par la production internationnale. Dans un deuxième temps, je voudrais faire voir comment le théâtre anglais a produit un schéma original qui va se perpétuer jusqu'à l'extrême fin du $\mathrm{XVI}^{e}$ siècle, où il coïncide même avec le théâtre public de l'Age d'Or élisabéthain.

Le théâtre anglais ne connaît ni farces, ni monologues, ni sotties. Est-ce à dire qu'il n'a aucune espèce de rapport avec ces types de pièces d'origine française ou espagnole ? Les pièces anglaises ont été décrites comme des moralités ou des interludes moraux. Nombreuses sont les définitions de la "moralité" anglaise qui la décrivent comme un théâtre moral et allégorique. Par "moral", on entend en général didactique, homilétique. G. R. Owst est même allé jusqu'à en faire un "sermon dramatisé"2. Quant au caractère allégorique, il n'est pas niable que la plupart de ces pièces contiennent des personnifications, et si nous suivons Werner Helmich qui écrit dans une très pénétrante étude sur la moralité française : «du moment où les personnifications agissent dans une fiction littéraire comme des êtres vivants, leur caractère allégorique ne peut être mis en doute» ${ }^{3}$, ces pièces seraient donc des allégories. Mais est-ce aussi simple que cela ? L'allégorie est-elle limitée à cette définition?

L'analyse allégorique n'est jamais d'une rigidité inflexible, ni d'une clarté parfaite, même dans l'interprétation du Texte Sacré, 
où on peut trouver trois ou quatre niveaux selon les cas, et où le sens allégorique se dispute quelquefois le deuxième niveau avec le sens moral ; le sens tropologique, ou moral, quant à lui, est en général logé au troisième niveau. Cette situation nous suggère que "moral" et "allégorique" ne sont peut-être pas des concepts totalement distincts. L'histoire littéraire elle-même nous ramène vers ce terme de "moral", puisque en Angleterre comme en France bien des pièces de théâtre sont désignées ainsi dans la deuxième moitié du $\mathrm{XVl}{ }^{\mathrm{e}}$ siècle. C"est sans doute que là le terme "moral" doit être entendu d'une manière moins étroite qu'aujourd'hui : non pas comme désignant la conduite désirable, mais de façon plus neutre et plus large, comme faisant référence au domaine de la philosophie morale, c'est-à-dire au micrososme humain, dans toutes ses capacités et en rapport avec les autres domaines, en particulier celui de la physique (qui traite du monde extérieur ou macrocosme), et celui du divin relevant de la théologie.

On a souvent avancé que la particularité de la moralité anglaise était de représenter le parcours complet de la vie de l'homme, de sa naissance à sa mort, sous forme d'une série de tentations qui mènent à la chute dans le péché, pour ensuite accéder au repentir et au salut. Ce type d'intrigue n'est pas limité à cette production nationale. Outre que ce parcours a été décrit dans d'innombrables traités, comme par exemple le Pélerinage de vie humaine de de Guilleville, si connu et maintes fois repris et traduit des deux côtés de la Manche, il est aussi présent dans le théâtre de langue française. Si on considère en effet un épisode de la Passion de Mons (1501), on y trouvera une séquence qui rappelle étrangement les moralités anglaises. Après la représentation de la chute des anges, de la création, de la tentation et de la mort d'Adam, et après la jubilation des démons en apprenant cette nouvelle (début très classique de mystère), un personnage de Humanus Genus apparaît, appelé ici Humain Lignaige, héros de moralité, qui interrompt les diables, se lamente sur sa propre mortalité et exprime sa peur d'aller en enfer. Les tentations d'Humain Lignaige, opérées par un certain nombre de Vices répètent sous une autre forme, les tentations infligées à Adam. Après cette interruption, le mystère et la moralité progressent, pour ainsi dire, parallèlement, puisque les événements qui font partie de l'histoire du monde - le Déluge, le sacrifice d'Isaac, la naissance du Christ, la Passion, la mise au tombeau, etc. - sont représentés à la manière habituelle des mystères; et à chaque épisode, Humain Lignaige réagit et le 
prologueur commente. L'ensemble montre les correspondances entre l'histoire religieuse du monde et la destinée de l'homme, jusqu'â la Résurrection du Christ, image du rachat, action qui figure la libération du péché, donc le salut de l'homme. Le Christ est alors personnification du Salut de l'homme et la Sagesse éternelle. On ne peut cependant pas le réduire au statut d'allégorie : est-ce que dans ce cas, le sens littéral n'est pas déjà le sens anagogique, dont le sens allégorique serait le sens tropologique? Plutôt que d'un jeu sur les niveaux allégoriques (sur lesquels en définitive tout le monde est d'accord), nous assistons plutôt à un jeu sur la représentation dramatique de ces concepts, à un effet de redondance à partir de la représentation linéaire habituelle des mystères, et qui fait bien comprendre aux lecteurs/spectateurs d'une autre époque ce qui était signifié par un récit fondateur des lois universelles régissant les rapports entre Dieu, l'homme et le monde.

Dans cette Passion, paraît non seulement Humain Lignaige, mais aussi le Monde et les vices, et la scène où Humain Lignaige s'en va "à l'ostel du Monde" précédé d'Orgueil, ("et va Orgueil le premier"), et où on lui donne de nouveaux habits, des habits mondains, à la grande satisfaction des diables, nous rappelle étrangement le début de la moralité de Nature de Henry Medwall, où le monde prend en charge l'homme naissant et lui donne son costume terrestre. L'homme devant les embûches du monde se retrouve aussi dans la moralité à quatre personnages (Chascun, Plusieurs, Le-Temps-qui-Court et le Monde, que Merle Fifield a appelée un "French Everyman"), et dans bien d'autres".

Les moralités hollandaise, les Spelen van Sinne, proposent aussi ce cheminement du personnage humain ; et là nous pensons au cas véritablement unique d'imbrication entre deux œuvres de provenances nationales différentes, le texte hollandais Elkerlijc, et l'anglais Everyman, au point qu'on a pu longtemps considérer cette traduction/adaption qu'est Everyman comme l'archétype même de la moralité anglaise. Certes en soi, le sujet d'Everyman n'a rien de surprenant pour une moralité anglaise (comme nous l'avons vu, le sujet est en fait international), mais la tonalité n'est pas particulièrement anglaise et surtout, la structure de Everyman porte le sceau de son origine étrangère.

Le thème, central dans ces pièces, de la lutte de l'homme contre les vices au cours de son itinéraire mondain est un thème européen. Il remonte à l'Ordo Virtutum de Hildegard de Bingen 
(circ.1150). Il se retrouve dans la pièce anglaise de Mind, Will and Understanding, or Wisdom who is Christ, où l'âme et ses trois "puissances" est (ou sont?) le(s) personnage(s) principal(aux). Mentionnons aussi le texte dialogué, parathéâtral de Thomas Chandler, Chancelier de l'Université d'Oxford, Le Liber Apologeticus de Omni Statu Humanae Naturae (circa 1460). Ces textes en latin apparaissent comme des symboles de cette culture internationnale du Moyen Age et de la Renaissance.

Je retiendrai particulièrement dans le Liber Apologeticus le motif, très répandu dans les Passions et les moralités françaises, du Procès de Paradis. S'il y en a un nombre assez réduit dans le corpus anglais (celui du Castle of Perseverance, du Ludus Coventriae et l'exemple imparfait de Respublica de N. Udall) les acteurs principaux, Justice et Miséricorde, se retrouvent fréquemment dans les interludes anglais.

Deuxième problème que nous pose l'existence de l'allégorie : l'allégorie est-elle cantonnée aux échange entre personnagespersonnifications (comme c'est le cas dans le procès de Paradis)? Ne peut-elle pas se lire aussi dans les textes réalistes et comiques? C'est ce que nous suggère J.-Ch. Payen à propos de la Farce du Cuvier ${ }^{5}$. Dans cette pièce, Jacquinot, mari martyrisé, demande à sa femme et à la mère de cette dernière, de lui rédiger un "rollet" de toutes les tâches qu'il doit exécuter, et puis quand le rollet est établi, sa femme tombe dans le cuvier; elle crie au secours, mais Jacquinot ne consent à l'en retirer, puisque ce travail n'est pas inscrit sur son rollet, que si elle accepte de renoncer à diriger le ménage. N'est-ce pas un monde à l'envers que représente ce ménage où domine la femme, un monde marqué du sceau du péché, et où l'eau du cuvier va figurer l'eau lustrale de la Rédemption qui ouvre la porte du monde racheté, celui où le mari est le maître ? N'est-il pas dit en effet, dans l'épilogue, que le sens va nous être donné "à mots couverts", c'est-à-dire, d'une certaine manière, par voie de métaphore ou d'allégorie ? Et on est tenté de suivre Payen lorsqu'il propose que le Cuvier est une "sorte de moralité" où "la norme conservatrice antiféministe exprime la volonté divine". Voilà donc le comique récupéré comme un élément constitutif de l'ordre fixe du monde, car il n'y figure qu'une subversion temporaire et superficielle. Cette vue semble cautionnée par l'antiquité du Carnaval et de la farce qui plongent leurs racines dans le plus haut Moyen Age, époque de rigueur doctrinale et de vision unifiée et totalisante. En faveur du sérieux potentiel de la farce, on pourrait citer dans le do- 
maine français, des Farces Moralisées, où peuvent apparaître des personnages allégoriques et où les propos sont moins crus et moins comiques que ceux de la farce habituelle.

S'il n'y a pas de farces anglaises, cela n'implique pas l'absence de dialogues farcesques; ils sont en effet inclus dans les interludes dits "moraux". L'interlude de Horestes (1567), met en scène l'histoire classique de la vengeance d'Oreste sur sa mère Clytemnestre. Or, de quoi sommes-nous témoins, lorsque le rideau se lève, (métaphoriquement parlant, s'entend) ? Un dialogue entre deux paysans (clowns), dont l'un accuse le chien de l'autre d'avoir tué son cochon, sur quoi le second accuse le premier d'avoir laissé le dit cochon saccager son potager ; et chaque paysan cherche à se venger du tort que lui a fait la bête de l'autre. Plus loin dans la pièce, deux soldats de l'armée d'Oreste, en réalité deux exemplaires du miles gloriosus, se lancent des défis démesurés et jamais honorés, et qui ont pour conséquence de tourner les vertus militaires en ridicule. Dans Horestes, comme dans plusieurs autres pièces connues sous le nom de "pièces hybrides" les éléments comiques (situations et personnages) viennent en contrepoint des passages sérieux, ce qui les investit d'un sens qui dépasse les grossièretés et les gesticulations dont ils sont apparamment composés. La conséquence de l'utilisation du mode allégorique permet donc d'investir de signifiance les parallélismes, puisque des manifestation superficielles dans des domaines différents et avec des effets opposés, peuvent être conçus comme procédant d'une origine commune.

Une autre conséquence de l'utilisation de ce mode est l'importance donnée au mot, au verbe, évidente dans tout les théâtres européens, le théâtre anglais y compris, qui véhiculent tous un neo-platonisme diffus. Un exemple, la farce française du Ramoneur de Cheminées, qui est fondée sur l'expression "ramoner la cheminée", dont, si vous ne le devinez déjà, vous allez rapidement comprendre le sens au résumé de l'intrigue. Un vieux ramoneur rentre chez lui, fatigué, et se lamente qu'on lui préfêre de jeunes et gaillards ramoneurs. Son jeune aide l'accable de sarcasmes devant sa jeune femme. Le ramoneur doit reconaître qu'il n'est plus bon à rien, et qu'il ne lui reste plus qu'à conseiller aux jeunes de "ramoner" pendant qu'il est encore temps. La pièce constitue donc une sorte de discours en circuit fermé, qui justifie a posteriori et en images l'existence de l'expression en question.

Dans une série d'interludes anglais, qu'on peut appeler "parémiologiques", la pièce n'est constituée que par un long com- 
mentaire sur l'expression de type proverbial qui lui sert de titre : AII for Money, Enough is as Good as a Feast, Like Will to Like. L'interlude progresse circulairement, là aussi, comme dans la farce du ramoneur.

On pourrait aussi évoquer comme preuve de cosmopolitisme la "banalité" des personnages et des épisodes mis en scène : Suzanne, Griselde, le reine Esther, Apius et Virginia, Cambise, Darius, l'Enfant Prodigue enfin, qui a une riche et double carrière, aussi bien du côté catholique que du côté protestant.

On pourrait aussi évoquer un autre type de circulation qu'est la traduction. Ainsi le théâtre tout entier de John Heywood est fortement inspiré de la farce française, en particulier John, John, qui est une très fidèle adaptation de la Farce du Pasté. Les sources s'entrecroisent souvent : pour une autre de ses pièces (Weather), J. Heywood s'inspire du portugais Gil Vicente, et dans une autre pièce encore (Witty and Witless) du dialogue français du Fol et du Sage. Le personnage de la Célestine, venu d'Espagne, est intégré dans un interlude anglais. Le fragment anglais de quelques lignes, connu sous le nom, de Somebody, Avarice and Minister a été identifié comme étant la traduction d'une moralité protestante française : La Vérité cachée.

Bref, tout ce théâtre donne l'impression, en dépit de modulations non négligeables dans les différents registres nationaux, d'une grande unité. Il constitue comme une grande famille, que Merle Fifield a qualifié, d'une expression très heureuse, «the community of morality plays».

Oui, il y a bien communité de thèmes et de traitement allégorique des sujets : mais, malgré ces ressemblances je perçois néanmoins une particularité insulaire qui se manifeste dans le domaine anglais. Pour en comprendre la spécificité, faisons un détour par les moralités hollandaises. Outre des contiguités géographiques et des similarités d'inspiration, le théâtre hollandais présente, il me semble, des caractéristiques structurelles qui nous mettent sur la voie d'une idiosyncrasie anglaise.

Mon premier exemple sera la pièce de Naaman, Prince de Syrie ${ }^{6}$. On peut lire dans la Bible (Rois, II,5), que Naaman, prince de Damas, fut converti par Elisé à la foi hébraïque après sa guérison de la lèpre dans les eaux du Jourdain. Au début de la pièce un personnage appelé Good Opinion (Vraie Doctrine) s'endort et rêve la pièce intérieure. Cette pièce intérieure se décompose à son tour en deux strates: les scènes de l'expédition de Naaman alternent 
avec des commentaires de la servante juive, Marthe, et de la princesse, épouse de Naaman, qui sont restées à Damas ; cette alternance donne, pour ainsi dire, du recul à l'événement. Dès que Naaman quitte la scène, le dormeur s'éveille, et demande le sens de cette histoire, qui lui est immédiatement fourni par les personnages eux-mêmes qui l'ont vécue : Naaman est le pêcheur, Marthe sa conscience, la Princesse l'Eglise ; et quand il demande où est le Jourdain purificateur, un rideau s'ouvre qui révèle un tableau - le toog -, du Christ en croix dont le sang s'écoule par les blessures, et au bas duquel est inscrite cette légende : «Voyez le Jourdain s'écoule des blessures de Notre Seigneur». On remarquera que la lecture allégorique, toujours possible dans le théâtre médiéval (ex. du Cuvier) est ici explicitée et exprimée par le parallélisme des scènes qui fournissent des perspectives différentes sur l'action, si bien que les personnages sont réalistes et allégoriques concurremment, d'une manière comparable à ceux du King John de J. Bale, et également par une structure en inclusions successives de pièces intérieures qui révèlent des couches différentes de signification.

Une leçon particulière nous est fournie par une autre pièce Hollandaise, Man's Desire and Fleeting Beauty ${ }^{7}$ Cette pièce est encadrée par le dialogue des citoyens de Gouda qui vont voir une pièce. La pièce intérieure est constituée par l'histoire de Désir Masculin qui convoite Beauté Passagère,-et obtient ses faveurs. Nos deux amants sont poussés dans les bras l'un de l'autre par deux personnages: Custom (que je traduirai probablement par Grégarisme ou Suivisme), et Fashion (Mode du jour). Ils sont laissés à leurs caresses amoureuses derrière un rideau, qui, lorsqu'il s'ouvre à nouveau, révèle un tableau - le toog - représentant le moment où la mort est sur le point de transpercer d'un coup de lance le couple enlacé. Ces deux personnages sont en marge de la situation principale ; ils ne participent pas de la même temporalité ; ils ne sont qu'à peine personnages dans la mesure où il ne leur arrive rien. Ils sont parodistes et commentateurs de l'action principale. Ils sont connus sous le nom générique de sinneken.

Ces sinneken nous mènent sur la voie d'un personnage propre aux interludes anglais : le Vice. Que le Vice soit central à la dramaturgie anglaise nous est suggéré par divers indices. Examinons à nouveau le cas de John Heywood. Même s'il n'est pas prouvé que toutes ses pièces sont inspirées d'œuvres françaises, l'influence est néanmoins évidente. Deux de ses pièces manifestement adaptées de modèles, The Play of love (1525) et The Play of 
Weather (1527), fournissent les plus anciens exemples d'un personnage nommé "Vice" explicitement mentionné dans la distribution. Ce personnage y est quelque peu plaqué sur l'action, et son addition dans une intrigue qui ne le comportait certainement pas dans la source, prouve, à cette date relativement ancienne (1525), la vitalité du personnage. Dans The Four PP et dans The Three JJ, qui sont des débats, genre attesté dans tous les pays d'Europe, Heywood a donné une place d'éminence à l'un des débatteurs, respectivement le colporteur ( $4 P P$ ) et James ( $3 J J$ ) qui structurent l'intrigue quasi inexistante, et alors qu'aucun personnnage n'a de rôle équivalent dans les débats français.

Autre exemple: plus loin dans le siècle (1578), Tom Tyler and Tom Taylor, qui est une véritable farce, possède pourtant un Vice nommé Désir.

Les pièces hybrides, relativement tardives, qui prennent comme intrigue un épisode "historique" (biblique, légendaire, etc.), à la manière d'un exemplum, possèdent toutes un Vice qui n'a aucun rapport avec l'épisode mis en scène.

Ces différents exemples, qui isolés seraient peu significatifs, fournissent des preuves convergentes de l'originalité de ce personnage qui constitue, je propose, le trait caractéristique, la preuve de l'insularité du théâtre anglais de la Renaissance. Son nom est mystérieux (a-t-il la même étymologie que "vice" l'opposé de la vertu ?), son histoire mal connue. Ce terme, peut-être en raison de son obscurité et de son mystère, n'évolue pas. Il ne se trouve pas non plus exclusivemntt au thêâtre ; on trouve aussi des traces de sa présence dans les fêtes populaires non-théâtrales.

Ce qui a dérouté bien des critiques du thêâtre Tudor est que cet emploi ne se manifeste pas toujours sous la forme d'un personnage. Il est bien plus essentiellement un principe organisateur protéen du commerce théâtral, qui peut investir plusieurs lieux successivement. Il est tour à tour le présentateur, le tentateur, le tenté, le comique satanique, l'indifférent, le porteur de nouvelles, le manipulateur et le commentateur-médiateur, confident de l'auditoire.

L'exemple le meilleur est peut-être le plus ancien : la pièce déjà citée de Wisdom. Cette pièce ne possède pas de Vice-personnage. Elle met en scène un diable, qui se dissimule bientôt sous les traits d'un galant, qui opère sous ce déguisement la séduction des trois parties de l'âme: Mind, Will et Understanding. Ces dernières deviennent à leur tour des émissaires du tentateur et des 
véhicules de la corruption jusqu'au moment du repentir. C'est successivement donc le Diable, le galant, les parties de l'àme qui mènent la danse, c'est-à-dire pratiquent l'action théâtrale dans son jeu et dans son rapport avec le spectateur; c'est à travers ces différents représentants - ou "acteurs" au sens sémiotique du terme - que court un principe unique nommé Vice.

Ben Jonson faisait référence, plus tard, après la disparition du théâtre "traditionnel", au "Vice Iniquity". Il croyait commte beaucoup l'on cru après lui, qu'il était un agent du mal et nécessairement un personnage. Certes ce n'est pas un auxiliaire du Bien. C'est une fonction dramatique qui transcende et nie ces catégories. Il n'est pas concerné par le schéma allégorique qui explique le théâtre médiéval en général. J'avancerai, pour ma part, qu'il ne doit pas être conçu comme un élément de sens, mais comme un mécanisme purement dramatique, qui fait partie de la forme. Le Vice est une métaphore théâtrale.

La pièce anglaise de tradition est en fait semblable à ses congénères européennes en ce qui concerne les thèmes et les sujets traités. Le ton qui y prévaut est tour à tour celui de la sottie, de la farce, de la moralité "à la française", de la vie de saint, de la chronique historique comme de la pièce romanesque. Mais la forme en est originale. le Vice médiateur lui donne une structure inconnue sur le "continent". Même s'il partage certains traits avec le badin français ou avec le gracioso espagnol, son rôle transcende ceux de ses collègues européens. C'est autour de lui que se constitue au

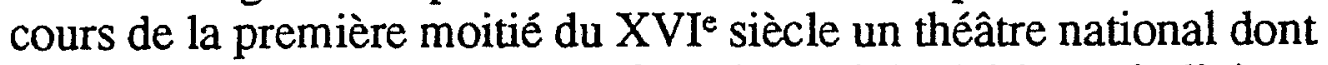
l'originalité est incontestable. C'est à ce riche héritage indigène, bien plus qu'aux pâles imitations de tragédies grecques, pratiquées dans les collèges et dans les Inns of Court, que devra sa miraculeuse éclosion le théâtre de l'Age d'Or élisabéthain.

Jean-Paul DEBAX

Université de Toulouse Le Mirail

NOTES

1. La Farce ou la machine à rire, Genève, Droz 1984, 24.

2. Literature and Pulpit in Medieval England, Londres, 1933, 526-45. Voir aussi : D.J.Leigh. "The Doomsday .Mystery Plays as an Eschatological Morality", Modern Philology, 67, 1970, 211-23. 
3. W. Helmich et Jeanne Wathelet-Willem. "La Moralité, genre dramatique à découvrir", Le Théâtre au Moyen-âge. Actes du $2^{\circ}$ colloque de la SITM, Alençon, juillet 1977, Ed. Aurore, Montréal, 1981, 20537.

4. "The Community of Morality Plays", Comparative Drama, 9, $1975,337$.

5. "La Farce du Cuvier, pièce allégorique ?", Revue d'Histoire du Théâtre, 3, 1973, 257-61.

6. Etude de deuxème main, car il n'existe pas de traduction en anglais, et je me fie à l'excellente analyse de W. M. H. Hummelen, "The Dramatic Structure of the Dutch morality Play", Dutch Crossing, $22,1984,17-26$.

7. Cette pièce a été traduite en anglais par A. Potter et E. Strietman, An offprint from Dutch Crossing, 1985. 